\title{
La dolarización en Ecuador: resultados macroeconómicos en las dos últimas décadas
}

\author{
Mauro Toscanini ${ }^{1}$, María Lapo-Maza ${ }^{1}$ y Miguel A. Bustamante ${ }^{1,2 \star}$ \\ (1) Universidad Católica de Santiago de Guayaquil. Av. Carlos Julio Arosemena Km. 11⁄2 vía Daule, Guayaquil, Ecuador. \\ (correo-e: mtoscanini@cu.ucsg.edu.ec; maria.lapo@cu.ucsg.edu.ec) \\ (2) Facultad de Economía y Negocios, Universidad de Talca. Talca, Chile. Dos Norte 685, 3465548 Talca, Chile. \\ (correo-e: mabu@utalca.cl)
}

* Autor a quien debe ser dirigida la correspondencia

Recibido Mar. 17, 2020; Aceptado May. 15, 2020; Versión final Jun. 18, 2020, Publicado Oct. 2020

\begin{abstract}
Resumen
El objetivo de este estudio fue revisar las ventajas y desventajas de la dolarización en la economía ecuatoriana mediante un análisis descriptivo de indicadores macroeconómicos clave. El diseño de la investigación fue de tipo no experimental longitudinal y descriptiva. Se identificaron fuentes oficiales para la recolección de indicadores económicos en el periodo 2000 a 2019 y se analizaron las tasas de crecimiento, inflación, desempleo y endeudamiento, además del saldo de la balanza comercial y presupuesto público. Los resultados evidencian que la dolarización estabiliza los precios manteniendo la inflación por debajo de un dígito. Sin embargo, el crecimiento económico de Ecuador no puede ser explicado tan sólo por esta causa, sino que debe ser explicado por otros factores, especialmente porque el país depende del petróleo, lo cual también se traduce en la necesidad de ejecutar ajustes recurrentes. Se concluye que la dolarización demanda políticas compatibles en concordancia con una disciplina fiscal severa.
\end{abstract}

Palabras clave: dolarización; indicadores macroeconómicos; política monetaria; inflación; desempleo

\section{Dollarization in Ecuador: a review of macroeconomic results in the last two decades}

\begin{abstract}
The objective of the present study was to review the advantages and disadvantages of dollarization in the Ecuadorian economy through a descriptive analysis of key macroeconomic indicators. The research design was non-experimental, longitudinal, and descriptive. Official sources were identified for the collection of economic indicators in the period 2000 to 2019. Growth rates, inflation, unemployment and indebtedness were analyzed, in addition to the balance of the trade balance and the public budget. The results showed that dollarization stabilizes prices by keeping inflation below one digit. However, Ecuador's economic growth can not be explained solely by this cause. It must be explained by other factors, especially the country's dependence on oil, which also translates into the need to make recurrent adjustments. It is concluded that dollarization demands compatible policies in accordance with severe fiscal discipline.
\end{abstract}

Keywords: dollarization; macroeconomic indicators; monetary policy; inflation; unemployment 


\section{INTRODUCCIÓN}

Tras la crisis económica y financiera que vivió Ecuador, conocida como feriado bancario y que se manifestó con fuerza a finales de la década de los años noventa, producto de las reformas en la legislación financiera ocurrida en el gobierno de Sixto Durán Ballén y Alberto Dahik, 1994, dejaron a los intermediarios financieros locales en absoluta libertad de manejar la cartera de crédito, libres de cumplir normas y controles. Esta situación dio paso, a la desinformación (Jensen y Mecking, 1976; Moreno, 2002), al pánico bancario, a problemas de agencia por las limitaciones de la banca de reserva fraccionada (Fama, 1980), a un desempleo galopante y a un descontrol de precios que derrumbó el poder adquisitivo, confirmando la teoría de la asimetría de información de los mercados (Edwards, 2001; Mochón, 2005) entre cuyas consecuencias se gestó un periodo de devaluación de la moneda nacional, el Sucre, con una escalada del tipo de cambio que sobrepasó la barrera de 20.000 Sucres por Dólar. A fines de 1999, los inversores habían ya retirado casi 6.000 millones de dólares, quebrado 16 instituciones financieras y originado un escenario en el cual, el entonces presidente Jamil Mahuad Witt, ratificado por su sucesor Gustavo Noboa Bejarano, obtuvo un financiamiento externo de 8.000 millones de dólares equivalentes a un 27\% del PIB (Lemoine, 2017), quedando obligado a dolarizar la economía ecuatoriana, lo que ejecutó anclando el tipo de cambio en 25.000 Sucres por Dólar americano (Banco Central de Ecuador, 2019).

La dolarización, definida por Dornbusch, (2001), como el proceso mediante el cual la moneda nacional de un país es reemplazada por el dólar como la unidad de medida de los precios, determina además los medios de pago, en este caso el dólar, regulando a su vez la tenencia de ahorros (Fama, 1980; Jensen y Mecking, 1976). Complementariamente, se define la dolarización como una evolución (Minda, 2005) donde el dólar es la reserva predominante de valor, unidad de cuenta y medio de intercambio, dando pie a diversas expresiones significativas tales como dolarización total, ocurrida en Ecuador y Panamá y dolarización parcial como es el caso de Perú (Edwards, 2001; Ponsot, 2003), mismas que distinguen el grado de uso de la moneda extranjera (Herrera y Caballero, 2002), avanzando en categorías de dolarización como dolarización no oficial, dolarización semioficial y dolarización oficial, última que convierte a la moneda extranjera en oficial con aprobación institucional.

La dolarización oficial sustituye la moneda nacional por una extranjera (Fama, 1980), reconociendo de esta manera el fracaso de la política interna, la pérdida de credibilidad y la incapacidad crónica para mejorar las finanzas públicas (Dornbusch, 2001; Minda, 2005), en consecuencia, un país decide dolarizar unilateralmente con el objetivo principal de restaurar la estabilidad monetaria y obtener credibilidad como país emisor, buscando superar la crisis de depreciación y deficiencias en el sistema bancario, entre otras (Herrera y Caballero, 2002; Jensen y Mecking, 1976). Para ello se introduce la moneda extranjera en los sistemas monetario, de producción e intercambio locales (Dornbusch, 2001) generando una serie de estímulos positivos y negativos de naturaleza económica y social. Sin perjuicio de ello, se identifican beneficios estabilizadores, aunque la dolarización oficial resulta costosa para las economías (Edwards, 2001), impactando los niveles de stock monetario (Moreno, 2002; Sierra y Lozano, 2010) y luego las tasas de inflación y de interés, niveles de actividad económica y de inversión, entre otras (Fama, 1980). Por lo tanto, la adopción de la dolarización oficial, por parte de una economía en desarrollo, requiere de un análisis profundo (Edwards, 2001) de contextos, causas y consecuencias diversas, partiendo con el cumplimiento de requisitos técnicos clave de equilibrio sectorial (Sierra y Lozano, 2010).

En este estado de cosas, Onur y Togay (2014), identifican ventajas y desventajas discutidas por Alesina y Barro (2001) y Calvo, (2001), quienes señalan como beneficio la eliminación significativa del sesgo inflacionario de la política monetaria, regulando significativamente la inflación (Edwards, 2001). Así mismo, Onur y Togay (2014) citan lo mencionando por Dornbusch (2001) quien señala que los países que se dolarizan tienden a crecer más rápido. Por otra parte Mántey (2013), señala a su vez que la adopción de una divisa dura reduce la vulnerabilidad económica, el riesgo devaluatorio y fortalece la credibilidad en las políticas financieras y monetarias y reduce los costos de transacción y acceso a los mercados de capitales internacionales (Guidotti y Rodríguez, 1992). En este contexto, la economía ecuatoriana que depende fuertemente de los precios del petróleo, se enfrentaría al hecho que una caída en los precios del crudo obligaría a reducir el gasto y la inversión así como a incrementar la contratación de deuda externa para mantener un ritmo adecuado de actividad económica (Dornbusch, 2001; Lemoine, 2017; (Minda, 2005) Salvia 2011) con la menor resistencia social que le sea posible conseguir (Salvia, 2017).

Por otro lado se afirma que la pérdida de señoreaje puede ser beneficiosa si obliga a un gobierno irresponsable a elegir políticas acertadas, aunque se reconoce que la dolarización afecta a los países pequeños a imponer un tipo de cambio fijo y reducir la competitividad, como le ocurre a Ecuador (Reyes, 2017), lo que se hace evidente cuando se reduce el saldo de la balanza comercial no petrolera. En 
consecuencia, la dolarización de Ecuador conlleva grados superiores de riesgo país alto, en tanto estimula políticas monetarias restrictivas y altas tasas de financiamiento externo. Ante este escenario, algunos estudios aseguran que un régimen monetario rígido es insostenible y de alto costo social (Keifman, 2004), que se traduce además en pérdida de señoreaje que implica perder la opción de la política monetaria aunque, en situaciones desfavorables, puede aliviar los ciclos económicos nacionales (Mántey, 2013; Minda, 2005). Por último y sobre la base de los conceptos sintéticamente desarrollados, el presente trabajo analiza las ventajas y desventajas de la dolarización de la economía ecuatoriana mediante el estudio descriptivo de algunos indicadores macroeconómicos clave.

\section{METODOLOGÍA}

El presente trabajo se realizó mediante la aplicación de diversos métodos de investigación. Entre estos se utilizó el método inductivo denominado también empírico, complementado con el deductivo que permite recurrir a teorías, análisis y principios generales destinados a explicar los casos particulares puestos en discusión (Hernández, et al., 2014), en este caso, aplicable a algunos países sudamericanos en contrastación (CEPAL, 2015; Lemoine, 2017). Sobre la base de lo indicado, se procedió a identificar algunas de las variables clave que determinan el comportamiento de las economías sostenidas en proposiciones, teorías y métodos de análisis, de carácter general, obtenidas del marco conceptual descrito como de la observación empírica de las variables macroeconómicas disponibles en los países considerados para alcanzar una conclusión general. Para ello se aplicaron algunas etapas de identificación, registro y análisis descriptivo de las variables económicas determinadas en este trabajo (Hernández, et al., 2014). De esta manera se optó examinar algunas de las variables que determinan el Producto Interno Bruto, PIB, tales como crecimiento económico, inflación, desempleo, presupuesto general del Estado y deuda pública. Para ello se aplicó el método analíticosintético (Hernández, et al., 2014), con la finalidad de mostrar relaciones y, a partir de ello, elaborar un juicio sintético y sistemático de los diferentes elementos heterogéneos y aislados que describen la situación de una economía (CEPAL, 2015; Mochón, 2005).

Desde la perspectiva instrumental, se procedió a utilizar algunas técnicas matemáticas y estadísticas orientadas a verificar y comprobar el comportamiento de las variables estudiadas (Hernández, et al., 2014), precisamente porque tienen impacto económico y social en los países objeto de comparación. Como lo señala el método behaviorista (Hernández, et al., 2014), se tuvo en consideración además que las variables inciden en forma directa e indirecta sobre las economías locales, lo que conlleva observar las políticas económicas, especialmente en referencia a las diversas estrategias que los gobiernos y el Estado ponen en práctica para conducir sus economías locales (Mochón, 2005). Estas políticas se ejecutan, por una parte, desde la política fiscal y por otra desde la política comercial que los gobiernos ponen en práctica, con el objetivo de conducir sus respectivos procesos económicos, por una parte a partir de la comprensión de sus propios ciclos económicos, sus finanzas públicas, el dinero, así como del crédito de la economía internacional, entre otras (Dornbusch, 2001; Hernández, et al., 2014).

De acuerdo con lo señalado, el presente trabajo se ocupa de observar el comportamiento de algunas de las variables macroeconómicas antes y después del proceso de dolarización (Hernández, et al., 2014). Se trata de un estudio de tipo descriptivo con diseño longitudinal. Los datos fueron obtenidos de fuentes secundarias oficiales tales como Banco Central de Ecuador (2019), Ministerio de Economía y Finanzas de Ecuador (2019), algunas estadísticas del Banco Mundial (2018) y de la Comisión Económica para América Latina y el Caribe (CEPAL, 2015; Lemoine, 2017). La inflación, fue medida a través del índice de precios al consumidor (IPC), la tasa de crecimiento del PIB se analizó mediante su valor nominal, el nivel de endeudamiento en sus valores oficiales, el presupuesto del Estado y deuda pública de acuerdo con los registros oficiales de las entidades pertinentes (Mochón, 2005). De manera adicional, se contrastaron algunos de estos indicadores con los de otros países, considerando aquellas parcialmente dolarizadas como ocurre con Perú y Panamá además de incluir otros países latinoamericanos, no dolarizados, pero de desempeño destacable que operan con sus respectivas monedas de circulación oficial como Colombia y Chile (CEPAL, 2015; Contraloría de la República, (2015).

\section{RESULTADOS}

A continuación se describen los resultados obtenidos del análisis del crecimiento económico, los índices de inflación, evolución del presupuesto general del Estado y deuda pública de los países contrastados.

\section{Crecimiento económico}

En Latinoamérica la dolarización se gestan en los procesos inflacionarios ocurridos desde los años setenta, como en Ecuador a fines de los 90' y que se asocia al fenómeno de Gresham, cuando la moneda "mala", se sustituye en las transacciones ordinarias por una divisa "buena" para lograr estabilidad (Guidotti y Rodríguez, 
1992). Dicha estabilidad ha de ocurrir en conjunto con decisiones y acciones complementarias, como lo es en Ecuador, una sustancial inversión pública producto de mayores ingresos petroleros provenientes de los altos precio del crudo y la renegociación de contratos petroleros, así como de ingentes recaudaciones tributarias derivados de las reformas tributarias que propiciaron en los últimos diez años una recuperación de la economía, la cual mostró tasas de crecimiento positivas, salvo en el año 2016 que decreció en -1,2\%, alcanzando sin embargo, un promedio del PIB real de 3,5\% durante los 20 años de dolarización (Figura 1).

Por otra parte, partiendo del hecho que la dolarización se hace sostenible cuando se responde a un decálogo de requisitos (Edwards, 2001) que exigen movilidad de factores, alto nivel de comercio de mercancías, diversificación productiva, precios y flexibilidad de salarios, entre otros (Sierra y Lozano, 2010), el análisis de la información estadística obtenida del Banco Central del Ecuador, 2019, permite apreciar que muchos de los requisitos del éxito se cumplieron, el PIB nominal que alcanzaba a 16.282 millones dólares el año 2000, pasó a un PIB de 45.503 millones el 2007 y a 107.562 millones el 2018. Haciendo evolucionar el ingreso per cápita de 1.300 dólares el año 2000 a más de 6.400 dólares el año 2019, logrando una tasa de crecimiento de 1,3\% el año 2018 y 0,1\% el año 2019 (Figura 1).

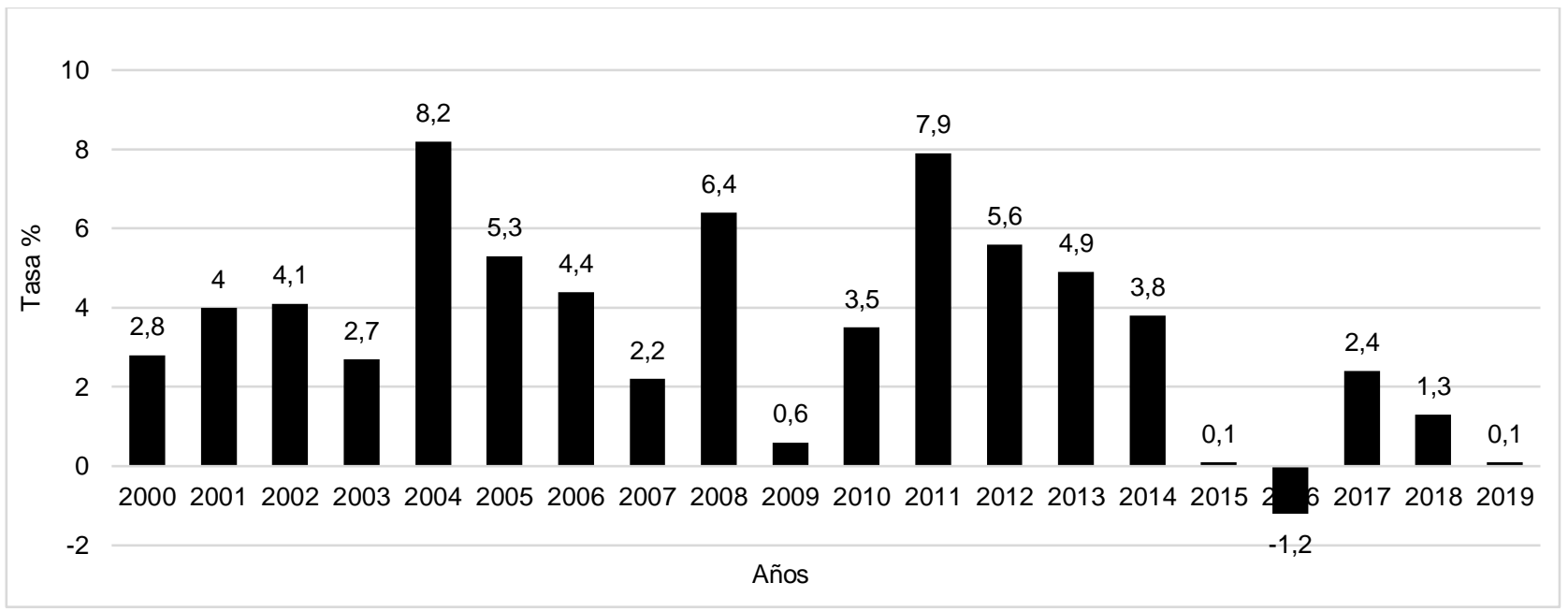

Fig. 1 Producto Interno Bruto Tasa de Variación Anual (Tasa \%)

Al comparar la tasa de crecimiento anual con otros países, según datos obtenidos del Banco Mundial (2018), se observa que el comportamiento en este indicador es similar entre Ecuador y el resto de los países latinoamericanos. Incluso en el año de recesión de 2008, la economía ecuatoriana se desempeñó igual que el resto de las economías contrastadas, aunque la tasa de crecimiento de Panamá se ubicó en la parte superior del rango observado. Resulta interesante concluir entonces, que le economía de Panamá, en la práctica dolarizada, sin Banco Central y sostenida en la norma constitucional de 1904 que no determina una moneda de curso forzoso, opera sobre una base estructural, sin presentar tasas significativamente más altas que las de los otros países analizados de la región, a excepción de los años 2007 y 2011 donde alcanza niveles superiores, como se aprecia en la Figura 2.

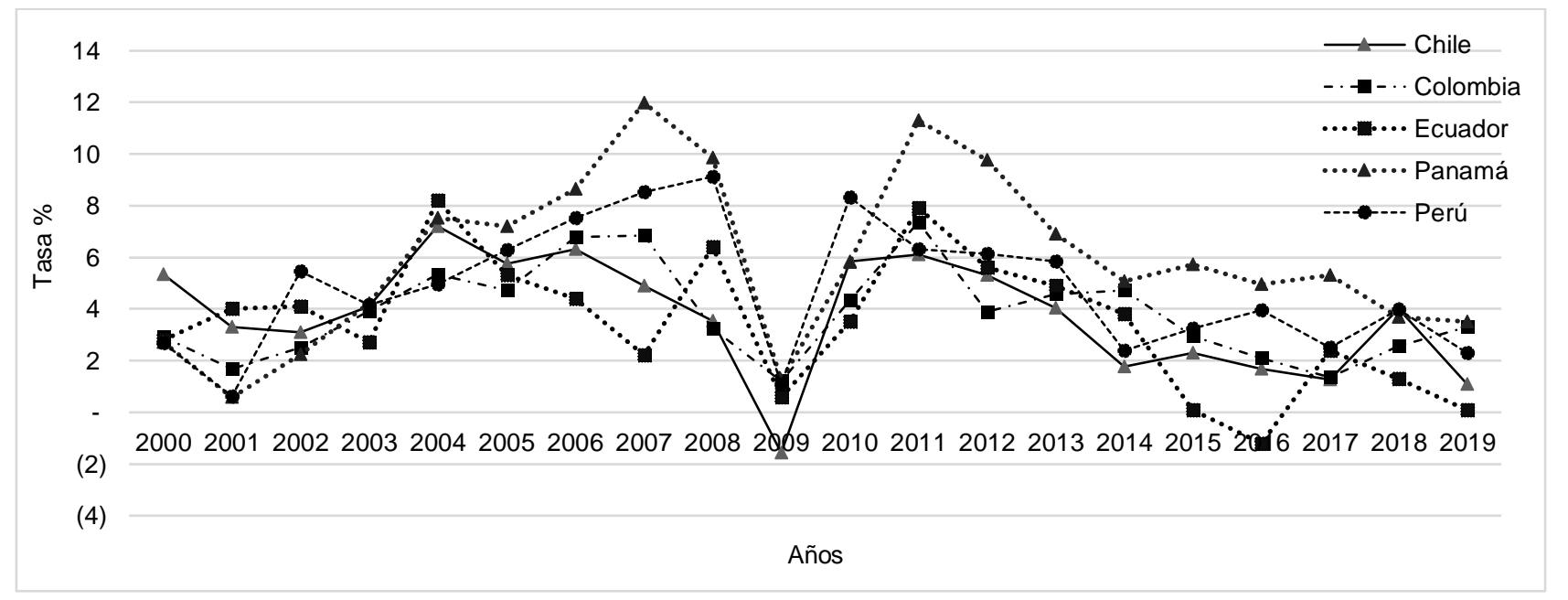

Fig. 2 Producto Interno Bruto Tasa de Variación Anual por Países (Tasa \%) 


\section{Inflación}

Ecuador enfrentó, una situación de cambio estructural en su relación comercial con los países aledaños (Colombia y Perú) que se explica, en parte, por el ajuste que hubo de asimilar a causa de una alta inestabilidad cambiaria previa a la dolarización que, como ya fue mencionado, generó devaluaciones más intensas que sus vecinos (Solórzano, et al, 2010. Dolarizar la economía implicó introducir la moneda extranjera para suplir la moneda doméstica, en este caso, el dólar estadounidense, reemplazando a la moneda local, el Sucre, lo cual estabilizó progresivamente las presiones inflacionarias, logrando como indica la Figura 3, que la inflación anual de $91,01 \%$ el año 2000 pasara $22,44 \%$ en 2001 y a partir del 2002, se redujera de ahí en adelante a tasas de un digito. En los últimos años la tasa de inflación anual se ubicó en 1,12\% el 2016, -0,20\% el 2017, $0,27 \%$ el 2018, llegando a 0,04\% el año 2019. Con todo, dolarizar tiene efectos (Alesina y Barro, 2001), sin perjuicio de ello, dolarizar o no las economías se enmarca, sin embargo, en un problema más general de elegir un determinado régimen cambiario, particularmente relevante en países en vías de desarrollo como Ecuador (Herrera y Caballero, 2002; Keifman, 2004; Paredes, 2017).

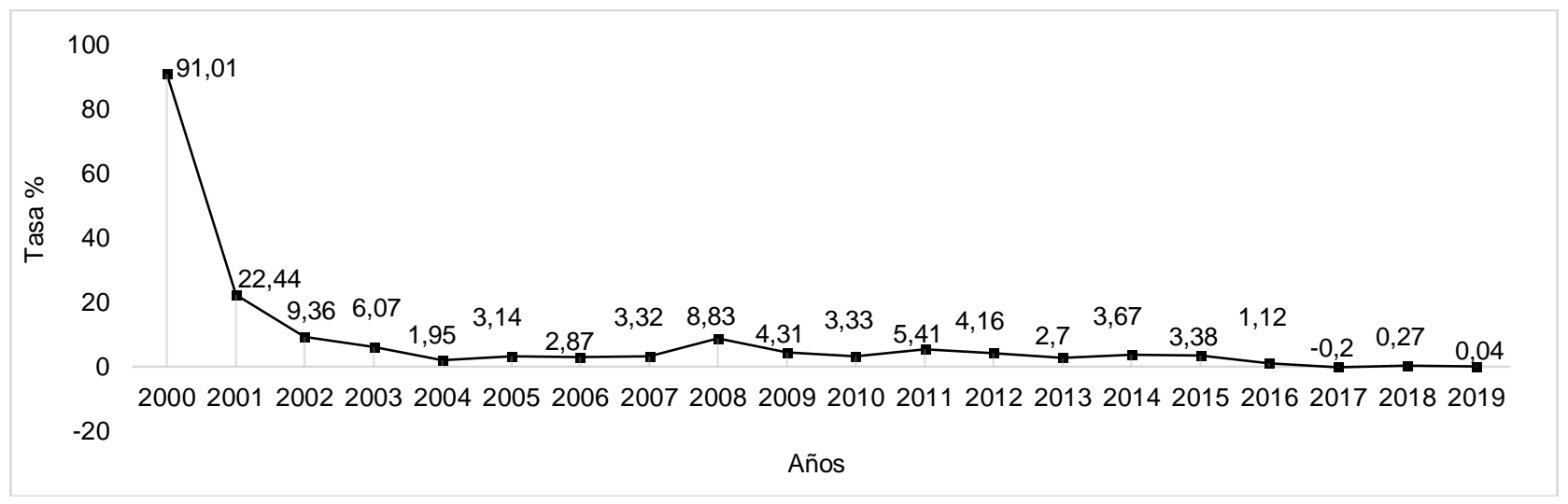

Fig. 3: Tasa de inflación anual del Ecuador (Tasa \%)

Por otra parte, al contrastar los resultados entre la inflación y desempleo (Figura 4), de acuerdo con datos obtenidos del Banco Mundial (2018), se observa que a partir del año 2001 el proceso de ajuste de la economía reduce la inflación de manera significativa generando una estabilidad evidente a excepción del año 2008, periodo en el cual sube levemente. Uno de los impactos de esta regulación inflacionaria se observa en el desempleo, el cual, frente a una inflación con tendencia a la baja, se mantiene en umbrales altos sobre el $10 \%$ entre los años 2000 a 2003 y en 2005 y 2006. Durante la recesión del año 2008, evidenciada en Ecuador el año 2009, los indicadores de inflación y desempleo reportan niveles similares (8\%). Los periodos de bonanza entre 2011 a 2015 muestran también estabilidad a niveles razonables. En promedio se registraron tasas de desempleo de 4,38\% e inflación de 3,86\% entre esos años. Posterior al año 2016 se evidenció el mismo patrón, con tasas de inflación que decae y desempleo que se eleva levemente.

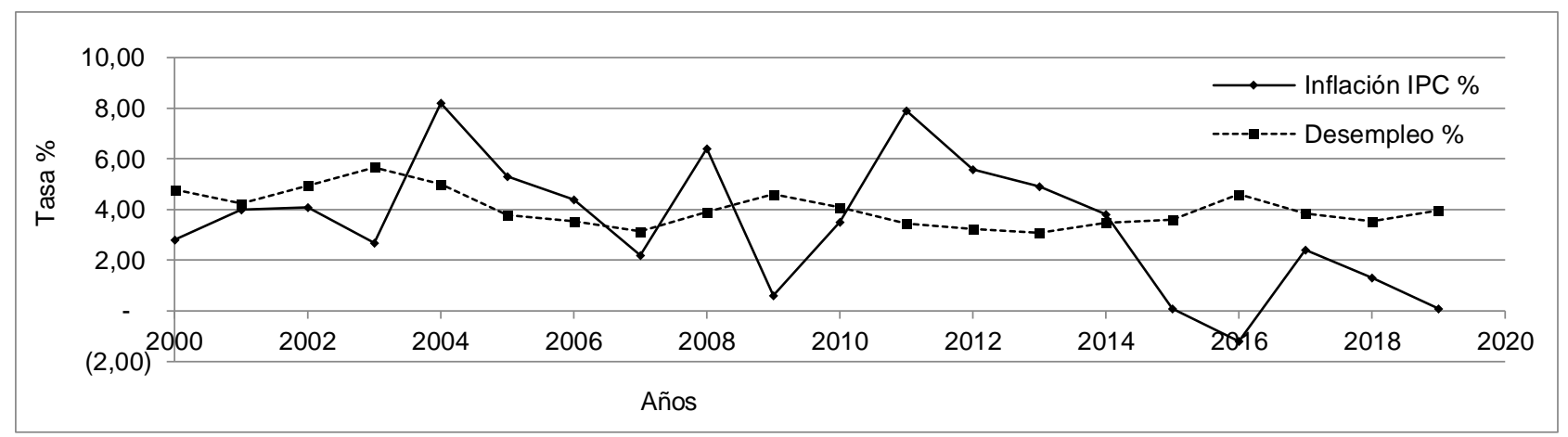

Fig. 4 Inflación y desempleo (Tasas \%)

Al contrastar la inflación de Ecuador con otros países de la región (Figura 5), de acuerdo con datos obtenidos del Banco Mundial (2018), se aprecia que no existen diferencias significativas con Colombia, Perú, Chile y Panamá. De hecho, en el año 2008, durante el periodo de crisis, Ecuador no logró mantener un nivel bajo como Colombia o Chile. Mientras que Panamá, cuya moneda corriente es el Balboa, cuenta con el dólar como una de las monedas más usadas y en los hechos como una de las economías dolarizadas, registra un nivel de inflación más bajo que Ecuador, pero que alcanzó niveles superiores a las tasas de inflación de Chile y Perú. 


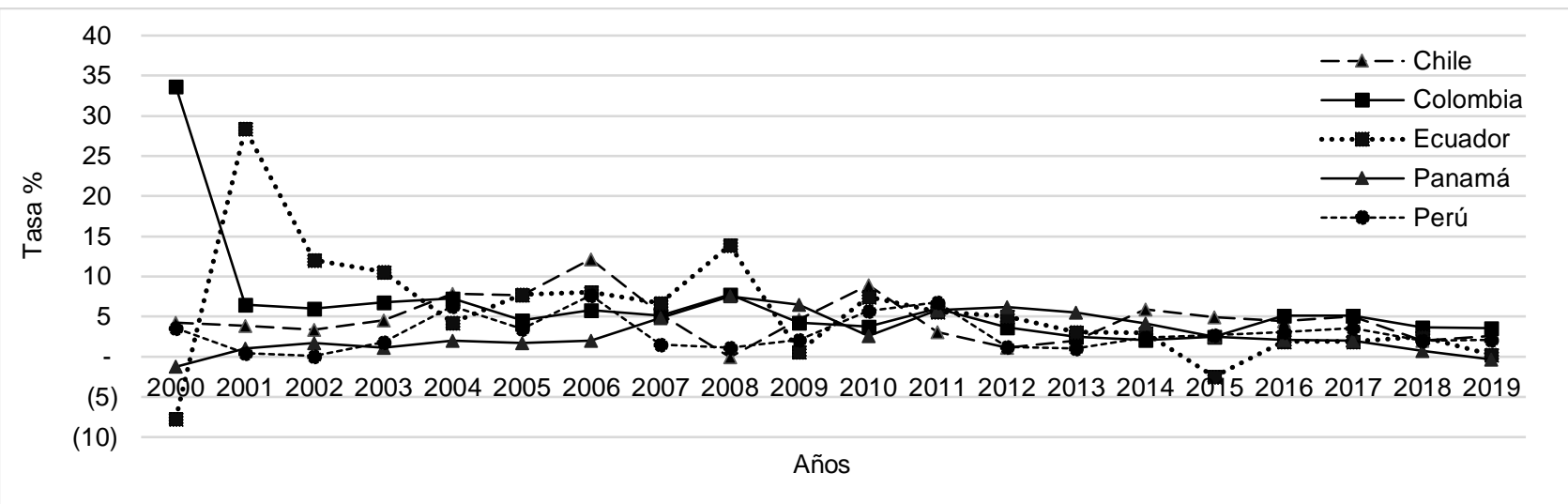

Fig. 5 Inflación anual por países (Tasas \%)

\section{Presupuesto General del Estado}

La evolución de las finanzas públicas de Ecuador en un período de 20 años, bajo gobiernos de distintas coaliciones políticas que totalizan 8 presidentes a la fecha, puede enmarcarse en dos etapas: Primero una fase de altos ingresos públicos, derivados de mayores ingresos petroleros por el incremento del precio internacional del crudo, renegociación de contratos petroleros y mayores recaudaciones tributarias, produjo en los primeros años bajos niveles de necesidades de financiamiento presupuestario, lo que permitió que se pudiera aumentar el gasto público y redujera el endeudamiento externo. Segundo, que luego de la caída del precio internacional del crudo, Ecuador inicia un proceso de menores ingresos fiscales, escalamiento del gasto público (Alesina y Barro, 2001; Minda, 2005; Paredes, 2017), mayores necesidades de financiamiento e incremento del endeudamiento público como se muestra en la Figura 6.

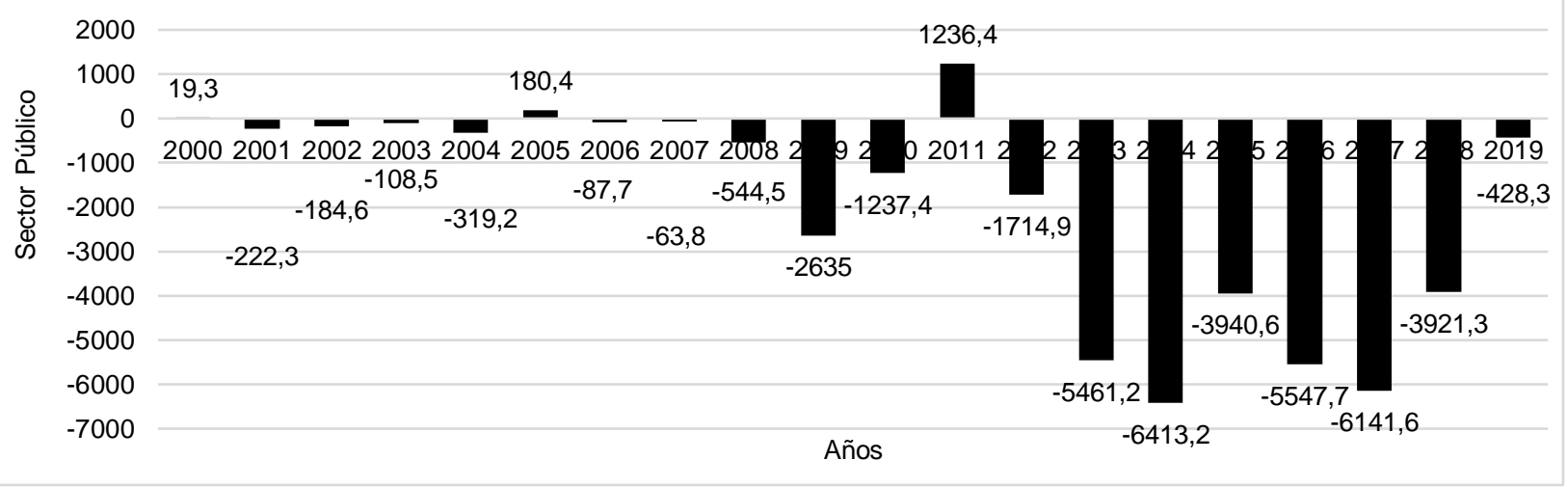

Fig. 1 Resultados de Presupuesto General del Estado 2000 - 2019

Por otra parte, de acuerdo con el Código Orgánico de Planificación y Finanzas Públicas, los egresos públicos permanentes y erogaciones por gastos corrientes, debieron ser financiados por ingresos públicos permanentes debido a la naturaleza de ambos. Mientras que los ingresos no permanentes, como el endeudamiento, se debieron asignar a actividades que incrementaren el capital. Sin embargo, también durante los gobiernos que van de 2007 a 2019, se ha dado un alto nivel de endeudamiento público buscando cubrir el déficit del presupuesto de cada año, esto a pesar de las medidas de ajustes como reducción de subsidios y disminución de gasto público, entre otros (Paredes, 2017).

\section{Deuda pública}

Desde una perspectiva general, la dolarización de una economía es una medida adecuada cuando las áreas territoriales e industrias están debidamente integradas a través del comercio internacional y el movimiento de factores es fuerte y flexible (Sierra y Lozano, 2010) lo cual genera riquezas y reporta tributos incrementales para resolver las inestabilidades (Minda, 2005). Sin embargo, cuando esto no ocurre con la debida fortaleza, las economías enfrentan tensiones como las que se muestran en la Tabla 1, que permite apreciar que durante el periodo analizado disminuyó el peso de la deuda pública en relación a un tamaño de la economía que creció progresivamente, pero que sin embargo, de acuerdo con lo informado en los Boletines Mensuales del Ministerio de Economía y Finanzas de Ecuador (2019), la deuda se ha seguido incrementando en los últimos años, sobrepasando el límite de 40\% del PIB establecido en el Código Orgánico de Planificación y Finanzas Públicas. 
Tabla 1: Endeudamiento Público Total en millones de USD.

\begin{tabular}{|c|c|c|c|c|c|c|c|}
\hline Período & $\begin{array}{l}\text { Saldo Deuda } \\
\text { Interna }\end{array}$ & $\begin{array}{l}\text { Saldo Deuda } \\
\text { Externa }\end{array}$ & Deuda Total & $P I B$ & $\begin{array}{l}\text { Deuda Interna / } \\
\text { PIB }\end{array}$ & $\begin{array}{l}\text { Deuda Externa / } \\
\text { PIB }\end{array}$ & $\begin{array}{l}\text { Deuda Total / } \\
\text { PIB }\end{array}$ \\
\hline & $A$ & $B$ & $C=A+B$ & $D$ & $A / D$ & $B / D$ & $C / D$ \\
\hline 2000 & $2.832,50$ & $11.335,40$ & $14.167,90$ & $18.318,60$ & $15,50 \%$ & $61,90 \%$ & $77,30 \%$ \\
\hline 2001 & $2.801,40$ & $11.372,80$ & $14.174,20$ & $24.468,30$ & $11,40 \%$ & $46,50 \%$ & $57,90 \%$ \\
\hline 2002 & $2.771,10$ & $11.388,10$ & $14.159,20$ & $28.548,90$ & $9,70 \%$ & $39,90 \%$ & $49,60 \%$ \\
\hline 2003 & $3.016,20$ & $11.482,30$ & $14.498,50$ & $32.432,90$ & $9,30 \%$ & $35,40 \%$ & $44,70 \%$ \\
\hline 2004 & $3.489,10$ & $11.060,40$ & $14.549,50$ & $36.591,60$ & $9,50 \%$ & $30,20 \%$ & $39,80 \%$ \\
\hline 2005 & $3.686,30$ & $10.850,30$ & $14.536,60$ & $41.507,00$ & $8,90 \%$ & $26,10 \%$ & $35,00 \%$ \\
\hline 2006 & $3.277,60$ & $10.214,90$ & $13.492,40$ & $46.802,00$ & $7,00 \%$ & $21,80 \%$ & $28,80 \%$ \\
\hline 2007 & $3.239,90$ & $10.632,70$ & $13.872,60$ & $51.007,80$ & $6,40 \%$ & $20,80 \%$ & $27,20 \%$ \\
\hline 2008 & $3.645,10$ & $10.088,90$ & $13.734,00$ & $61.762,60$ & $5,90 \%$ & $16,30 \%$ & $22,20 \%$ \\
\hline 2009 & $2.842,20$ & $7.389,20$ & $10.231,40$ & $62.519,70$ & $4,50 \%$ & $11,80 \%$ & $16,40 \%$ \\
\hline 2010 & $4.665,00$ & $8.671,70$ & $13.336,70$ & $69.555,40$ & $6,70 \%$ & $12,50 \%$ & $19,20 \%$ \\
\hline 2011 & $4.506,50$ & $10.046,40$ & $14.552,90$ & $79.779,80$ & $5,60 \%$ & $12,60 \%$ & $18,20 \%$ \\
\hline 2012 & $7.780,50$ & $10.871,80$ & $18.652,30$ & $87.498,60$ & $8,90 \%$ & $12,40 \%$ & $21,30 \%$ \\
\hline 2013 & $9.926,60$ & $12.913,90$ & $22.840,50$ & $95.129,70$ & $10,40 \%$ & $13,60 \%$ & $24,00 \%$ \\
\hline 2014 & $12.558,30$ & $17.581,90$ & $30.140,20$ & $101.726,30$ & $12,30 \%$ & $17,30 \%$ & $29,60 \%$ \\
\hline 2015 & $12.546,00$ & $20.205,90$ & $32.751,90$ & $99.290,40$ & $12,60 \%$ & $20,40 \%$ & $33,00 \%$ \\
\hline 2016 & $12.457,40$ & $25.522,80$ & $37.980,20$ & $99.937,70$ & $12,50 \%$ & $25,50 \%$ & $38,00 \%$ \\
\hline 2017 & $14.785,70$ & $31.749,80$ & $46.535,50$ & $104.295,90$ & $14,20 \%$ & $30,40 \%$ & $44,60 \%$ \\
\hline 2018 & $13.733,70$ & $35.695,20$ & $49.428,90$ & $108.398,10$ & $12,70 \%$ & $32,90 \%$ & $45,60 \%$ \\
\hline ene-19 & $17.132,00$ & $38.681,90$ & $55.813,90$ & $109.134,50$ & $15,70 \%$ & $35,40 \%$ & $51,10 \%$ \\
\hline feb-19 & $16.819,10$ & $38.534,30$ & $55.353,40$ & $109.134,50$ & $15,40 \%$ & $35,30 \%$ & $50,70 \%$ \\
\hline mar-19 & $17.113,60$ & $38.914,20$ & $56.027,80$ & $109.134,50$ & $15,70 \%$ & $35,70 \%$ & $51,30 \%$ \\
\hline abr-19 & $17.154,90$ & $38.643,30$ & $55.798,20$ & $109.134,50$ & $15,70 \%$ & $35,40 \%$ & $51,10 \%$ \\
\hline may-19 & $16.636,70$ & $39.081,20$ & $55.717,90$ & $109.134,50$ & $15,20 \%$ & $35,80 \%$ & $51,10 \%$ \\
\hline jun-19 & $16.626,20$ & $39.279,30$ & $55.905,50$ & $109.134,50$ & $15,20 \%$ & $36,00 \%$ & $51,20 \%$ \\
\hline jul-19 & $16.910,80$ & $38.737,30$ & $55.648,10$ & $109.134,50$ & $15,50 \%$ & $35,50 \%$ & $51,00 \%$ \\
\hline ago-19 & $16.490,90$ & $39.238,50$ & $55.729,40$ & $109.134,50$ & $15,10 \%$ & $36,00 \%$ & $51,10 \%$ \\
\hline sep-19 & $16.161,40$ & $40.767,50$ & $56.928,90$ & $109.134,50$ & $14,80 \%$ & $37,40 \%$ & $52,20 \%$ \\
\hline oct-19 & $16.090,53$ & $40.841,35$ & $56.931,88$ & $107.435,67$ & $14,98 \%$ & $38,01 \%$ & $52,99 \%$ \\
\hline nov-19 & $15.991,98$ & $40.791,01$ & $56.782,99$ & $107.435,67$ & $14,89 \%$ & $37,97 \%$ & $52,85 \%$ \\
\hline dic-19 & $15.843,64$ & $41.495,58$ & $57.339,22$ & $107.435,67$ & $14,75 \%$ & $38,62 \%$ & $53,37 \%$ \\
\hline 2019 & $15.843,64$ & $41.495,58$ & $57.339,22$ & $107.435,67$ & $14,75 \%$ & $38,62 \%$ & $53,37 \%$ \\
\hline
\end{tabular}

En general se observa que la opción de dolarizar la economía se ha utilizado para solucionar desequilibrios macroeconómicos graves tales como, brotes inflacionarios, tipos de cambio desordenados, desequilibrios fiscales y en cuenta corriente además de desempleo, entre otros de similar naturaleza apreciados en economías como la Argentina (Sierra y Lozano, 2010), sin embargo, también pudieron ser apreciadas el año 2000 en Ecuador con una deuda pública total de 14.167 millones de dólares (77,3\% del PIB) que, al año 2017 se ubicó en 46.535 millones de dólares (44,6\% del PIB) (Lemoine, 2017). Esa deuda adquirida en los últimos años es cara, del orden de un 10\%, como es el caso de la deuda que se mantiene con la economía China, cuyo servicio de la deuda equivalente a un $10 \%$ del PIB, al $50 \%$ de las exportaciones, alcanza la tercera parte del presupuesto general del estado (Banco Mundial, 2019). 
La dolarización de las economías en desarrollo no es una invención reciente (Moreno, 2002) trae beneficios y exigencias. Se inicia en ocasiones con una dolarización semioficial buscando la estabilidad de precios mediante la convertibilidad (Keifman, 2004; Minda, 2005) pretendiendo por una parte, contrarresta la amenaza de la inflación que trae consigo la devaluación y por otra, el acuerdo social en torno a la política de dolarización, sin embargo, variables tales como disciplina, capacidad de reacción sectorial y apremios impositivos, inciden sobre otras variables de gestión pública, situación que, en Ecuador, en este último gobierno, durante estos dos últimos años (2018-2019), ha incrementado el endeudamiento y ha seguido siendo una constante en las decisiones para financiar el presupuesto del Estado, persistiendo en consecuencia, desequilibrios fiscales y un alto endeudamiento público que, a septiembre de 2019, llega a 56.928 millones de dólares $(52,2 \%$ del PIB), de manera que, en menos de dos años totaliza más de 10 mil millones de dólares sobrepasando el estándar nacional de endeudamiento público (Ministerio de Economía y Finanzas de Ecuador, 2019).

\section{DISCUSIÓN}

El análisis del PIB ecuatoriano que se aprecia en la Figura 1, muestra un comportamiento altamente volátil marcando las tasas más altas entre los años 2004 y 2011, los cuales a causa de la dolarización, son calificados como un beneficio por Dornbusch (2001), pero que sin embargo, no se sostienen por sí mismos porque la dolarización es una herramienta de último recurso (Fama, 1980) y por otra parte, porque el crecimiento del PIB depende a su vez de la conjunción de otras variables como el gasto público que se incrementa fuertemente a contar del año 2008, alcanzando su cúspide el año 2014, como se muestra en la Figura 6. Previo a la dolarización, la devaluación permitía mejorar la competitividad, pero a costa de un ajuste automático via reducción de salarios reales (Paredes, 2017), situación que genera resistencias sindicales y sociales, que bloquean el equilibrio, anteponiéndose a leyes de "flexibilización laboral" como fue evidente en la economía Argentina (Piva, 2013). Sin embargo, en el caso de Ecuador, precisamente a causa de la dolarización el país incrementa su riqueza promedio (Dornbusch, 2001) incrementó el ingreso per cápita en 4,9 veces durante el periodo incidiendo sobre variables macroeconómicas clave como inflación y desempleo (Edwards, 2001). Complementariamente, desde una perspectiva teórica, se aprecia en general que quienes tiende a apoyar la dolarización, como es el caso de la banca privada o la devaluación como ocurre con la industria y la construcción, pasan previamente por la convertibilidad, por supuesto si con ello se obtiene el sostén de un proyecto de dolarización que fortalezca sus crecimientos (Guidotti y Rodríguez, 1992; Moreno, 2002)

Teniendo en consideración que la devaluación tiene efectos negativos de gran magnitud sobre las economías cambiando los acervos y afectando las decisiones sectoriales de inversión (Mántey, 2013) se puede ver que los sectores de la banca, la industria y la construcción valoran positivamente la dolarización, puesto que incide sobre el crecimiento (Figura 5). Se observa que los países dolarizados, Ecuador oficialmente y Panamá por la práctica monetaria cotidiana, derivan en una política monetaria flexible, seguido de Perú parcialmente dolarizado y los restantes que no lo están (Chile y Colombia), que resultan más duramente afectados por el costo financiero de la deuda que los países dolarizados (Edwards, 2001; Ponsot, 2003; Moreno, 2002), sin embargo, dados los esfuerzos gubernamentales de dichos países que implementan políticas públicas compatibles (Dornbusch, 2001; Minda, 2005), logran una política monetaria coherente con la industrial, educativa, tecnológica, fiscal y crediticia, entre otras, permitiéndoles una inserción más favorable en la economía global, mostrándolos como más exitosos, sin apreciarse diferencias significativas entre ellos (Edwards, 2001).

Del análisis del endeudamiento público de la Tabla 1, se observan contextos, causas, consecuencias, mitos y realidades diversas (Edwards, 2001), razón por la cual, efectivamente, la política del Gobierno no está determinada por los equipos técnicos, sino por las constricciones, restricciones y oportunidades que les presenta la realidad (Moreno, 2002; Salvia 2011), en consecuencia, el Estado ecuatoriano, sin perjuicio del esfuerzo de sus equipos, llegó progresivamente al límite del endeudamiento permitido en las reglas macrofiscales (Onur y Togay, 2014) confirmando las apreciaciones de pérdida del señoreaje, sin embargo, se espera que a futuro se logren los beneficios derivados de la dolarización de una economía en desarrollo (Dornbusch, 2001) tales como estabilidad fiscal, fomento productivo, atracción de inversiones y generación de empleo. Sin embargo, es preciso reconocer que el desarrollo financiero influye positivamente en el volumen de las exportaciones, pero que puede verse contrarrestado por el endeudamiento, ejerciendo un fuerte efecto negativo que anula el efecto positivo del tipo de cambio en la competitividad (Berman y Berthou 2009). Sintetizando, el modelo económico ecuatoriano cambió progresivamente de uno basado en pequeños desfases fiscales entre 2000 y 2008, desfases identificados por la teoría (Dornbusch, 2001) a uno basado en mayor gasto público y déficits presupuestarios permanentes referenciados por Onur y Togay (2014) y que se califican como desventajas por la pérdida del señoreaje provocando efectos adversos como los califica Reyes (2017), sin embargo, el desafío país es relevante (Keifman, 2004), es preciso aliviar los efectos sobre los ciclos económicos, si se quiere finalmente recoger los beneficios de la dolarización (Mántey, 2013; Onur y Togay, 2014) en la economía del país. 
En general, se aprecia que la dolarización implementada en la economía ecuatoriana impactó generando una tendencia a la baja en la inflación como se aprecia en la Figura 3, lo cual a su vez impactó positivamente en la tasa de desempleo que también tendió a decrecer como lo muestra la Figura 4, todo lo cual beneficia de manera relevante a la economía ecuatoriana confirmando que los éxitos tempranos se manifiestan a través de la eliminación o mitigación del riesgo cambiario, lo cual estabiliza las tendencias naturales a la especulación en el tipo de cambio y mejora las percepciones de certeza de las inversiones de mayor plazo. Con todo, las certezas no son homogéneas, puesto que pueden generar disputas empresariales, entre quienes apoyan las devaluaciones y quienes pugnan por la dolarización, produciendo un resquebrajamiento del consenso con las reformas económicas implementadas (Berman y Berthou 2009; Piva, 2013; Salvia, 2017) y que ha sido observadas en Argentina, donde se entrecruzan los sectores financiero e industrial que presionan por políticas económicas que les sean más convenientes (Salvia, 2017).

Consecuente con lo señalado, se ratifica la eficacia de la dolarización oficial y total implementada por Ecuador según los postulados de Ponsot, (2003). Al analizar las tasas de inflación y desempleo en el periodo $2001-$ 2018 (Figura 4) se observa, que la economía dolarizada ecuatoriana sobre todo en los últimos años, muestra un proceso de ajuste típico en estos casos de acuerdo con los planteamientos de Onur y Tugay, (2004) que se hacen evidentes cuando en la economía se estabiliza el ciclo de recesión, se estabiliza el crecimiento en tasas positivas sostenibles, lo cual regula el efecto de la inflación, calificándolos como positivos de acuerdo con los planteamientos de Fama, (1980), sin perjuicio de lo cual la dolarización impulsa también un alza relativa del desempleo, calificado éste como efecto negativo (Minda, 2005).

Por último, es preciso señalar que las devaluaciones generan incertidumbre, respecto a la evolución futura del tipo de cambio y la inflación, afectando negativamente la dinámica exportadora de los países (Berman y Berthou 2009; Mántey, 2013). Es por ello que, la comparación de los niveles de inflación progresivamente regulados por la dolarización (Alesina y Barro, 2001; Calvo, 2001) en la Figura 3, permite apreciar que, en cuatro de estos países no se observan diferencias significativas (Colombia, Perú, Chile y Panamá) y que Ecuador, si bien aplica dolarización oficial (Fama, 1980), le resulta costosa por tener que enfrentar un periodo inicial con tasas inflacionarias altas, especialmente el año 2008, pero que le permitió obtener los beneficios previstos por Onur y Togay (2014) y discutida por Alesina y Barro (2001) y Calvo, (2001), que se manifiestan a contar del año 2009, en un incremento del crecimiento global y per cápita, una baja progresiva de la inflación y una reducción sostenida del desempleo, a ubicando al país en el promedio de los países analizados.

\section{CONCLUSIONES}

Tras el análisis realizado a lo largo del presente trabajo se concluye que efectivamente se lograron identificar ventajas y desventajas en el proceso de dolarización en Ecuador. Entre estas, se logró incrementar el crecimiento, contener y reducir la inflación e impactar positivamente la moneda estabilizando las tendencias, proveyendo certezas a la inversión de mayor plazo así como ratificando los beneficios de la dolarización oficial implementada por Ecuador. En este contexto, se lograron identificar incidencias adversas a causa de las intervenciones de política pública, que si bien estabilizaron la fase de recesión el orden y confianza en el gobierno y el control de la inflación, como efectos positivos, produjo efectos negativos, como el incrementó de la deuda pública a causa de la pérdida de disciplina fiscal por parte de la autoridad económica y política.

Al comparar los efectos de la dolarización de los cinco países analizados se concluye que sus comportamientos fueron bastante similares, ascendiendo y declinando juntos a lo largo de los años analizados, sin embargo, en el caso de Ecuador, los efectos observados resultaron ser claramente más exacerbados, eventualmente a causa de los beneficios de la dolarización implementada como por los impactos negativos derivados de la pérdida de disciplina fiscal apreciada en las tasas crecientes de endeudamiento alcanzado en la segunda mitad del periodo analizado.

Se concluye así mismo que la dolarización genera en Ecuador periodos de volatilidad alta en las tasas de crecimiento del PIB, logrando incrementar sin embargo la riqueza per cápita de largo plazo. Es por ello que, contrastados estos hallazgos entre los cinco países analizados se aprecia que dos de ellos, Ecuador y Panamá, están dolarizados formalmente el primero y por la práctica monetaria el segundo, muestran resultados diferentes y hasta opuestos. Se observa así mismo que Perú, que se encuentra parcialmente dolarizado además de Chile y Colombia que no lo están, presentan resultados singulares a causa de la diversidad de políticas públicas, disciplina fiscal y regulaciones implementadas por los respectivos Estados.

Ecuador muestra como resultados adversos el haber superado los límites de gasto público cuya consecuencia ha sido el endeudamiento. Por el contrario, muestra tasas de desempleo afortunadamente decrecientes, finalmente, se puede afirmar que la realidad económica de Ecuador cambió progresivamente pasando desde un modelo económico que enfrentaba pequeños desfases fiscales hacia un modelo que busca capitalizar los beneficios estabilizadores de la dolarización. 


\section{REFERENCIAS}

Alesina, A., y Barro, R. J., Dollarization. American Economic Review, 91(2), 381-385 (2001)

Banco Central de Ecuador Boletín Macroeconómico (2019)

Banco Mundial, Global Waves of Debt (2019).

Berman, N. y Berthou, A., Financial Market Imperfections and the Impact of Exchange Rate Movements on Exports, https://doi.org/10.1111/j.1467-9396.2008.00781.x Review of International Economics, vol. 17, núm. 1, Malden Mass., Wiley Blackwell, pp. 103-20 (2009)

Calvo, G. A., Capital Markets and the Exchange Rate, with Special Reference to the Dollarization Debate in Latin America. Journal of Money, Credit and Banking, 312-334 (2001)

CEPAL Comisión Económica para América Latina y el Caribe Estudio Económico de América Latina y el Caribe 2015. Santiago de Chile: Naciones Unidas (2015)

Contraloría de la República. Informe sobre la situación de las Finanzas del Estado 2014. Bogotá. (2015).

Dornbusch, R., Exchange Rates and the Choice of Monetary-Policy Regimes. Fewer monies, Better Monies. American Economic Review, 91(2), 238-242 (2001)

Edwards, S., Dollarization-Myths and Realities. Journal of Policy Modeling, 23(3), 249-265 (2001)

Fama E., Agency Ploblems and the Theory of the Firm, Journal of Political Económy, Vol. 88, No. 2 (Apr., 1980), pp. 288$307(1980)$

Guidotti, P. y Rodríguez, C. A., Dollarization in Latin America: Gresham's Law in Reverse? IMF Staff Papers, vol. 39, issue 3, 518-544 (1992)

Hernández, R., Fernández, C. y Baptista, P., Metodología de la Investigación (Sexta ed.). México: Mc Graw Hill (2014)

Herrera V., B. y Caballero B., J., Dolarizar: ¿Realmente un Dilema? Cuadernos de Economía No. 36, 101 - 152 Bogotá (2002).

Jensen R. y Mecking W., Theory of the Firm: Managerial Behavior, Agency cost and Ownership Structure. Journal of financial economics $N^{\circ} 3,305-360$ (1976)

Keifman, S., Auge y Derrumbe de la Convertibilidad Argentina: Lecciones para Ecuador. https://doi.org/10.17141/iconos.19.2004.27 Revista de Ciencias Sociales, Íconos, № 19 25-34 (2004)

Lemoine, M., Coup Dur Pour la fin de Cycle en Equateur. Mémoire des luttes, 04 avril, (2017)

Mántey, G., ¿Conviene Flexibilizar el tipo de Cambio para Mejorar la Competitividad? Revista Problemas del Desarrollo, 175 (44), octubre-diciembre (2013)

Minda, A., Full Dollarization: A last Resort Solution to Financial Instability in Emerging Countries?. The European Journal of Development Research, 17(2), 289-316 (2005)

Ministerio de Economía y Finanzas Estadísticas Fiscales. Información presupuestaria (2019)

Mochón M., F., Cap. 5. Una Visión Global: la Macroeconomía. En F. Mochón, Economía, Teoría y Política (Quinta ed., págs. 240-241). Madrid: Mc Graw Hill (2005)

Moreno R., Á., Dolarización en América Latina: una Mirada no Convencional. Cuad. Econ. vol.21 no.36 Bogotá Jan./June (2002).

Onur T., B. K. y Togay, S., Efectos de la Dolarización Oficial en una Pequeña Economía Abierta: el Caso de Ecuador. Investigación Económica, LXXIII (290), 51-86 (2014)

Paredes, G. J., Ecuador:¿por qué Salir de la dolarización? Revista CEPAL №121 149-167 (2017)

Piva, A. Acumulación y Hegemonía en la Argentina Menemista. Buenos Aires: Biblos (2013)

Ponsot J. F., La Dollarisation: une Interpre'tation Institutionnaliste et Macroe'conomique'. Forum de la Régulation, 9-10 October, Paris (2003)

Reyes, A., Crecimiento de la Economía Ecuatoriana: Efectos de la Balanza Comercial no Petrolera y de la Dolarización. Revista Espacios, 38(61) 14-29 (2017)

Salvia S., Alianzas de los Empresarios Industriales en la Crisis del Neoliberalismo en Argentina. https://doi.org/10.1590/1678-987317256204 Revista de Sociología Politica, v. 25, n. 62, p. 93-113, jun. (2017)

Salvia, S., El país en riesgo. Crisis Financiera y Gestión Neoliberal en Argentina (1999-2001 Estudios - Centro de Estudios Avanzados, núm. (26) 111-124. (2011)

Sierra, L. P. y Lozano, D. M., ¿Qué Sabemos Sobre la Dolarización y sus Efectos en las Economías Latinoamericanas que la Adoptaron?* Revista Facultas de Ciencias Económicas, Vol. XVIII (1), 119-132 Junio (2010)

Solórzano, G., Rivadeneyra, I. y Guamán, L. Á., Cambio Estructural en la Competitividad Ecuatoriana Después de la Dolarización. Revista de Economía del Caribe № 6. 71-94 (2010). 